\title{
Análise Comparativa de Abordagens de Associação entre os Estilos de Aprendizagem de Felder-Silverman e os Metadados do Padrão IEEE LOM
}

\author{
Janderson J. B. Aguiar', Joseana M. Fechine', Evandro B. Costa² \\ ${ }^{1}$ Universidade Federal de Campina Grande (UFCG), Campina Grande - PB - Brasil \\ ${ }^{2}$ Universidade Federal de Alagoas (UFAL), Maceió - AL - Brasil \\ janderson@copin.ufcg.edu.br, joseana@dsc.ufcg.edu.br, evandro@ic.ufal.br
}

\begin{abstract}
Learning Styles (LS) e Learning Objects (LO) are terms increasingly found in research on Computers in Education. These concepts can be combined to optimize the search and use of $L O$ in repositories, based on the student's LS. In this paper, we present a comparative analysis of two recent researches that performed associations between the attributes of the LO (based on the IEEE LOM standard) and LS (based on the Felder-Silverman theory). The purpose of this paper is to expose that some aspects of these associations are not trivial, and there are approaches with significant differences; therefore more studies concerning these associations need to be performed.
\end{abstract}

Resumo. Estilos de Aprendizagem (EA) e Objetos de Aprendizagem (OA) são termos cada vez mais encontrados em pesquisas relativas à Informática na Educação. Esses conceitos podem ser associados para, por exemplo, otimizar a busca e utilização dos OA em repositórios, com base nos EA do aluno. Neste artigo, é apresentada uma análise comparativa de duas pesquisas recentes que realizaram associações entre os atributos dos $O A$, baseados no padrão IEEE LOM, e os EA, baseados na teoria de Felder-Silverman. O objetivo deste artigo é expor que alguns aspectos dessas associações não são triviais, havendo abordagens com diferenças significativas e que, portanto, mais estudos relativos a essas associações precisam ser realizados.

\section{Introdução}

De acordo com Braga et al. (2014), a existência de repositórios de Objetos de Aprendizagem (OA) e a ampliação do acesso das escolas a computadores/internet embora ainda sem a qualidade ideal —, têm proporcionado um cenário de uso de OA nas aulas. Estudos relativos a OA são, portanto, cada vez mais relevantes no contexto de Informática na Educação (IE), uma vez que tais recursos estão relacionados à aprendizagem dos alunos.

Além disso, é importante destacar que o processo de aprendizagem não é vivenciado por todos os indivíduos da mesma forma, sendo desenvolvidos Estilos de Aprendizagem (EA) que enfatizam algumas habilidades sobre as outras (KOLB, 1984).

Uma vez que os alunos possuem diferentes EA, é interessante desenvolver OA com características diferenciadas, descrevendo essas características por meio de variados metadados, que são descritores - com diversas informações, a exemplo de 
data de criação e formato - utilizados para facilitar a classificação e busca dos OA, permitindo sua utilização em diversas plataformas.

Nesse contexto, percebe-se que, além de bastante relevantes na área de IE, os conceitos de OA e EA são intrinsecamente relacionados.

Há algumas pesquisas que realizaram associações entre as categorias de padrões de OA e modelos teóricos de EA, tais como Oliveira (2013), Resende et al. (2014), Carvalho e Dorça (2014) e Carvalho et al. (2014). Apesar de alguns aspectos se assemelharem, há algumas diferenças significativas nessas pesquisas em relação às características dos OA que se adaptam melhor aos EA. Neste trabalho, é apresentada uma análise baseada nos estudos de Oliveira (2013) e de Carvalho et al. (2014), identificando similaridades e diferenças entre as abordagens adotadas, além de reflexões sobre a importância desse tipo de associação para pesquisas futuras em IE.

Embora as pesquisas citadas anteriormente sejam relacionadas à pesquisa descrita neste artigo, não foram encontrados trabalhos cujo foco tenha sido uma análise visando comparar abordagens de associação entre EA e atributos de OA, especialmente com enfoque nas dimensões de EA do modelo de Felder-Silverman (FELDER e SILVERMAN, 1988) e nos atributos (metadados) do padrão IEEE LOM (IEEE, 2002).

O "acesso participativo e universal do cidadão brasileiro ao conhecimento" foi apontado pela Sociedade Brasileira de Computação (SBC) como um dos grandes desafios científicos da computação para a década 2006-2016 (SBC, 2006). Para tratar tal desafio, é necessária "uma interlocução com outras áreas de conhecimento, como as Ciências Humanas e Sociais, cuja prática e discurso científicos diferem daqueles próprios da Ciência da Computação" (SBC, 2006).

Este artigo, por meio da análise comparativa de abordagens de associação entre metadados de OA e dimensões de EA, visa contribuir para a potencialização do uso das tecnologias em prol da aprendizagem, e, consequentemente, contribuir com reflexões relativas ao desafio comentado no parágrafo anterior.

A metodologia para esta análise comparativa consistiu em identificar os metadados utilizados dos OA em cada uma das duas abordagens/pesquisas brasileiras anteriormente mencionadas, verificando como cada um desses metadados foi associado, pelos autores dessas pesquisas, às dimensões de EA.

$\mathrm{Na}$ seção 2, são abordados alguns conceitos importantes para esta pesquisa, relativos a EA e OA. Na seção 3, é descrita a comparação realizada. Na seção 4, são realizadas algumas reflexões e considerações finais sobre a importância desse tipo de estudo para pesquisas futuras em IE.

\section{Pressupostos teóricos}

Esta seção destina-se a apresentar os fundamentos teóricos referentes a Objetos de Aprendizagem (OA) e Estilos de Aprendizagem (EA), indispensáveis para compreender a comparação realizada, foco deste artigo.

\subsection{Objetos de Aprendizagem}

Objeto de Aprendizagem pode ser definido como uma entidade, digital ou não digital, que pode ser utilizada, reutilizada ou referenciada para o ensino, educação ou 
treinamento assistidos pela tecnologia, como sistemas de Educação a Distância e ambientes colaborativos de ensino (IEEE, 2002).

Com o crescente interesse na tecnologia de OA, existe na Web um grande número de repositórios de OA, a exemplo do ARIADNE (http://ariadne-eu.org/) e MERLOT (http://merlot.org). Os repositórios correspondem a bancos de dados que armazenam metadados, referências (URL) e, em alguns casos, armazenam os próprios OA (MACHION, 2007).

Dentre os padrões de metadados existentes, há o IEEE LOM (Learning Object Metadata) (IEEE, 2002). Este padrão é amplamente aceito no meio acadêmico, sendo considerado um modelo completo, apresentando um conjunto de metadados organizados em nove categorias, permitindo a fácil inserção de novas categorias e elementos em categorias existentes (VICARI et al., 2010).

Os metadados do padrão IEEE LOM são agrupados nestas categorias: Geral (informações gerais do OA), Ciclo de vida (características relativas ao histórico e ao estado atual do OA), Meta-metadados (metadados utilizados para descrever os metadados do OA), Técnica (requisitos e características técnicas do OA), Educacional (características educacionais e pedagógicas do OA), Direitos (direitos de propriedade intelectual e condições de uso do OA), Relação (características que definem o relacionamento entre um OA e outros), Anotação (comentários sobre o uso do OA) e Classificação (descreve o objeto de acordo com algum sistema de classificação prédefinido) (IEEE, 2002; VAZ, 2009).

Dentre os metadados da categoria Técnica, está o Formato. Na categoria Educacional, há o Tipo de Interatividade, o Tipo de Recurso de Aprendizagem, o Nível de Interatividade, a Densidade Semântica, a Dificuldade, entre outros. Dentre os metadados da categoria Geral, há os metadados Estrutura e Nível de Agregação. Esses metadados citados são utilizados na comparação explanada na seção 3 , uma vez que foram utilizados em pelo menos uma das pesquisas analisadas.

\subsection{Estilos de Aprendizagem}

Os Estilos de Aprendizagem (EA), de acordo com Riding e Rayner (2000), consistem no modo preferido, individual e habitual com que os alunos organizam e representam novas informações durante o processo de aprendizagem.

Dentre os modelos de EA utilizados em diversas áreas ${ }^{1}$, o modelo de FelderSilverman (FELDER e SILVERMAN, 1988) é o mais utilizado em pesquisas relacionadas à adaptação e personalização de materiais de aprendizagem, tanto em nível nacional (AGUIAR, FECHINE e COSTA, 2014) quanto internacional (VALASKI, MALUCELLI e REINEHR, 2011).

Segundo Felder e Silverman (1988), os EA referem-se às maneiras pelas quais os indivíduos preferem receber e processar as informações. Ao receber a informação externa (captada pelos sentidos) e a informação interna (que surge introspectivamente), o indivíduo seleciona o material a ser processado. Ao processar, tal indivíduo pode usar simples memorização ou raciocínio indutivo ou dedutivo, reflexão ou ação, introspecção

\footnotetext{
${ }^{1}$ Coffield et al. (2004) realizaram uma revisão da literatura e identificaram 71 modelos de EA.
} 
ou interação com outros indivíduos. Como resultado, o conteúdo abordado no material selecionado é aprendido de uma forma ou de outra ou, então, não é aprendido.

O modelo de Felder-Silverman (FELDER e SILVERMAN, 1988; FELDER, 2002) contempla quatro dimensões de EA: (i) Dimensão Processamento, com os estilos Ativo e Reflexivo; (ii) Dimensão Percepção, com os estilos Sensorial e Intuitivo; (iii) Dimensão Entrada, com os estilos Visual e Verbal; e (iv) Dimensão Compreensão, com os estilos Sequencial e Global.

Os indivíduos predominantemente Ativos aprendem, em geral, por meio da experimentação ativa, e compreendem as informações mais eficientemente discutindo e aplicando os conceitos; por outro lado, os predominantemente Reflexivos necessitam de um tempo sozinhos para pensar/refletir sobre as informações obtidas. Aqueles com predomínio do estilo Sensorial geralmente preferem lidar com situações concretas, dados e experimentos; por outro lado, aqueles com predomínio do estilo Intuitivo são inovadores, gostam de lidar com abstrações, conceitos e teorias. Os indivíduos predominantemente Visuais aprendem mais facilmente por meio de demonstrações, diagramas, figuras, filmes, fluxogramas; por outro lado, os que apresentam predomínio do estilo Verbal compreendem melhor as informações transmitidas por meio de palavras. Os indivíduos Sequenciais aprendem geralmente melhor quando o conceito é expresso de maneira contínua de dificuldade e complexidade; por outro lado, os indivíduos Globais são, em geral, multidisciplinares, aprendendo em grandes saltos e lidando com os conteúdos de forma aleatória (FELDER e SILVERMAN, 1988; DIAS, GASPARINI e KEMCZINSK, 2009).

\section{Comparação de abordagens de associação entre dimensões de EA e metadados de OA}

Em estudos relativos à adaptação e personalização de materiais de aprendizagem, alguns pesquisadores realizaram associações entre as categorias de padrões de OA e modelos teóricos de EA. Dentre os mais recentes, e considerando o contexto brasileiro, é possível citar estudos como o de Oliveira (2013), o de Resende et al. (2014), o de Carvalho e Dorça (2014) e o de Carvalho et al. (2014). Nesses trabalhos, são associados os metadados (atributos) das categorias do padrão IEEE LOM às dimensões de EA do modelo de Felder-Silverman.

Apesar de alguns aspectos se assemelharem nas abordagens de associação de Carvalho et al. (2014) - que expande as abordagens de Resende et al. (2014) e de Carvalho e Dorça (2014) - e Oliveira (2013), há, todavia, diferenças significativas ao definir as características dos OA que se adaptam melhor aos EA. No Quadro 1, é possível observar simplificadamente as diferenças entre esses quatro trabalhos. Nesta seção, é apresentada uma análise baseada nos estudos de Oliveira (2013) e Carvalho et al. (2014), identificando similaridades e diferenças entre as abordagens adotadas.

Segundo Oliveira (2013), o atributo Formato — da categoria Técnica - e os atributos Tipo de Interatividade, Tipo de Recurso de Aprendizagem, Nível de Interatividade, Densidade Semântica e Dificuldade — da categoria Educacional detalham os OA e permitem uma associação com os EA.

No estudo de Carvalho et al. (2014), além dos atributos considerados por Oliveira (2013), são considerados os atributos Estrutura e Nível de Agregação — da categoria Geral - para associar os OA aos EA. 
Uma vez que adotaram abordagens diferenciadas ao realizarem as associações, foram constrastadas as tabelas (com os pesos) da pesquisa de Oliveira (2013) com as regras (no formato Prolog) da pesquisa de Carvalho et al. (2014)². É possível afirmar que as associações se assemelham nos aspectos tratados na subseção 3.1. Por outro lado, as diferenças entre essas pesquisas, visualizadas superficialmente no Quadro 1, são tratadas na subseção 3.2.

Quadro 1. Breve comparação entre as pesquisas de Oliveira (2013) [0], Resende et al. (2014) [R], Carvalho e Dorça (2014) [D] e Carvalho et al. (2014) [C] sobre a associação de EA e atributos de OA.

\begin{tabular}{|c|c|c|c|c|c|}
\hline & \multicolumn{4}{|c|}{ Dimensões do Modelo de EA de Felder-Silverman } \\
\hline & & Processamento & Percepção & Entrada & Compreensão \\
\hline \multirow{8}{*}{ 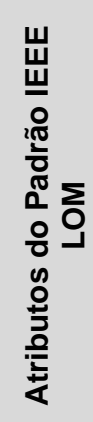 } & Formato & $\mathrm{O}|\mathrm{R}| \mathrm{D} \mid \mathrm{C}$ & $\mathrm{O}|\mathrm{R}| \mathrm{D} \mid \mathrm{C}$ & $\mathrm{O}|\mathrm{R}| \mathrm{D} \mid \mathrm{C}$ & $\mathrm{O}$ \\
\hline & Tipo de Interatividade & $\mathrm{O}|\mathrm{R}| \mathrm{D} \mid \mathrm{C}$ & $O|R| D \mid C$ & & 0 \\
\hline & $\begin{array}{l}\text { Tipo de Recurso de } \\
\text { Aprendizagem }\end{array}$ & $\mathrm{O}|\mathrm{R}| \mathrm{D} \mid \mathrm{C}$ & $R|D| C$ & $\mathrm{O}|\mathrm{R}| \mathrm{D} \mid \mathrm{C}$ & C \\
\hline & Nível de Interatividade & $\mathrm{O} \mid \mathrm{C}$ & O & & O \\
\hline & Densidade Semântica & & 0 & & $C$ \\
\hline & Dificuldade & & $\mathrm{O}|\mathrm{R}| \mathrm{D} \mid \mathrm{C}$ & & \\
\hline & Estrutura & & & & $R|D| C$ \\
\hline & Nível de Agregação & & & & C \\
\hline
\end{tabular}

\subsection{Semelhanças entre as abordagens de associação}

Em relação ao atributo Formato, há três situações semelhantes nas associações de Oliveira (2013) e Carvalho et al. (2014): (i) em relação à dimensão Processamento, o formato vídeo favorece o estilo Reflexivo; (ii) em relação à dimensão Percepção, os formatos vídeo, imagem, áudio e texto favorecem o estilo Sensorial; (iii) em relação à dimensão Entrada, os formatos vídeo e imagem favorecem o estilo Visual, enquanto que os formatos áudio e texto favorecem o estilo Verbal.

Em relação ao atributo Tipo de Interatividade, o tipo expositivo indica um processamento mais Reflexivo, enquanto o tipo ativo de interatividade indica processamento Ativo da informação; além disso, uma interatividade expositiva favorece a percepção Sensorial.

Em relação ao atributo Tipo de Recurso de Aprendizagem, há duas situações: (i) em relação à dimensão Processamento, os tipos de recurso simulação, exercício e experimento favorecem o estilo Ativo; (ii) em relação à dimensão Entrada, os tipos de recurso diagrama, gráfico, figura e slide favorecem o estilo Visual, enquanto que os tipos de recurso narrativa, palestra e índice favorecem o estilo Verbal.

Em relação ao atributo Nível de Interatividade, níveis altos favorecem o processamento mais Ativo. Em relação ao atributo Dificuldade, maior grau de dificuldade associada ao $\mathrm{OA}$ favorece o estilo Intuitivo e menor favorece o estilo Sensorial.

\footnotetext{
${ }^{2}$ Devido à limitação de espaço, as tabelas/regras dos trabalhos comparados não foram reproduzidas neste artigo. Todavia, na seção de Referências, há os hiperlinks para o acesso integral a esses trabalhos.
} 


\subsection{Diferenças entre as abordagens de associação}

A primeira diferença entre essas abordagens consiste na maneira de associação realizada - Oliveira (2013) fez uso de pesos em tabelas, e Carvalho et al. (2014) definiram regras em Prolog, como citado anteriormente. Mesmo assim, foi possível analisar as impressões de cada autor em sua respectiva abordagem e, por conseguinte, foram observados os seguintes aspectos contrastantes:

- Em relação ao atributo Formato:

○ Oliveira (2013), em relação à dimensão Processamento, considerou que, além de vídeo, o formato imagem favorece o estilo Reflexivo; em relação à dimensão Entrada, considerou que o formato vídeo favorece apenas o estilo Visual; em relação à dimensão Compreensão, considerou que os formatos vídeo, imagem, áudio e texto favorecem o estilo Sequencial.

- Carvalho et al. (2014), em relação à dimensão Processamento, consideraram que, além de vídeo, os formatos áudio e texto/html favorecem o estilo Reflexivo; em relação à dimensão Entrada, consideraram que o formato vídeo favorece tanto o estilo Visual quanto o estilo Verbal; em relação à dimensão Compreensão, não realizaram associação com esse atributo.

- Em relação ao atributo Tipo de Interatividade:

- Oliveira (2013) considerou que há relação com a dimensão Compreensão (devido à interatividade expositiva ter maior probabilidade da informação estar de forma encadeada e lógica - estilo Sequencial).

- Carvalho et al. (2014) não realizaram associação desse atributo com a dimensão Compreensão.

- Em relação ao atributo Tipo de Recurso de Aprendizagem:

- Oliveira (2013), em relação à dimensão Processamento, considerou que os tipos de recurso exame, enunciado de problema, questionário e autoavaliação, além de exercício, favorecem o estilo Reflexivo, enquanto que os tipos de recurso tabela, índice, gráfico, diagrama, figura, narrativa e palestra favorecem o estilo Ativo; em relação à dimensão Entrada, considerou que slide e tabela favorecem principalmente o estilo Visual; em relação às dimensões Percepção e Compreensão, não realizou associação com esse atributo.

- Carvalho et al. (2014), em relação à dimensão Processamento, consideraram que os tipos de recurso exame, enunciado de problema e questionário favorecem o estilo Ativo, enquanto que os tipos de recurso tabela, índice, gráfico e slide favorecem o estilo Reflexivo; em relação à dimensão Entrada, consideraram que os tipos de recurso tabela, exame e experimento favorecem o estilo Verbal; em relação à dimensão Percepção, consideraram que os tipos de recurso diagrama, gráfico, figura, narrativa, palestra, índice e slide favorecem o estilo Sensorial, enquanto que os tipos de recurso tabela e auto-avaliação favorecem o estilo Intuitivo; em relação à dimensão Compreensão, consideraram que o tipo de recurso experimento favorece o estilo Sequencial, enquanto que o tipo de recurso tabela favorece o estilo Global. 
- Em relação ao atributo Nível de Interatividade:

- Oliveira (2013) considerou que há relação com as dimensões Percepção (quanto maior esse nível, mais favorecido é o estilo Intuitivo) e Compreensão (nível baixo favorece o estilo Sequencial por haver maior probabilidade da informação estar de forma encadeada e lógica).

- Carvalho et al. (2014) não realizaram associações desse atributo às dimensões Percepção e Compreensão.

- Em relação ao atributo Densidade Semântica:

- Oliveira (2013) considerou que há relação com a dimensão Percepção: alta densidade - com menos detalhes sobre o assunto no OA — é mais próxima ao estilo Intuitivo, enquanto que baixa densidade é mais próxima ao estilo Sensorial.

- Carvalho et al. (2014) consideraram que há relação com a dimensão Compreensão: alta densidade é mais próxima ao estilo Global, enquanto que baixa densidade é mais próxima ao estilo Sequencial.

- Em relação ao atributo Estrutura:

- Oliveira (2013) não relacionou tal atributo com as dimensões de EA.

- Carvalho et al. (2014) consideraram, em geral, que a Estrutura rede é relacionada ao estilo Global, enquanto que a linear é relacionada ao estilo Sequencial.

- Em relação ao atributo Nível de Agregação:

- Oliveira (2013) não relacionou tal atributo com as dimensões de EA.

- Carvalho et al. (2014) consideraram que os valores 2,3 e 4 são relacionados ao estilo Global, e o valor 1 ao estilo Sequencial ${ }^{3}$.

\section{Considerações Finais}

É importante cada vez mais a utilização do conceito de EA em pesquisas voltadas a IE para que os estudantes não sejam tratados de forma igual em relação ao modo como adquirem conhecimento. Com o passar dos anos, a quantidade de OA disponíveis em repositórios tende a crescer, além de haver a necessidade constante de prover experiências de aprendizagem motivadoras para os alunos, tais como a adaptatividade de ambientes e a recomendação de $\mathrm{OA}$ mais próximos às suas preferências e particularidades.

Um dos desafios atuais nas pesquisas em IE é a criação de sistemas adaptativos e modelos eficientes para recomendar conteúdo, visando melhorar a aprendizagem dos alunos, especialmente considerando que estes possuem modos diferentes de aprender. Há muitas propostas de Sistemas de Recomendação (SR) de OA considerando abordagens clássicas de SR, entretanto, muitas vezes desconsiderando os EA dos aprendizes na construção do perfil do usuário. É importante, pois, analisar estudos sobre como associar os atributos dos OA aos EA.

\footnotetext{
${ }^{3}$ No trabalho de CARVALHO et al. (2014), os níveis de agregação 2, 3 e 4 estão relacionados tanto ao estilo Global quanto ao estilo Sequencial. A partir do contato via e-mail com os autores, foi repassada esta correção: "Na verdade, os Níveis 2, 3 e 4 são mapeados para o estilo Global, e 1 para o estilo Sequencial!" (CARVALHO, 2015).
} 
A análise comparativa das abordagens explanada na seção 3 aponta para a necessidade de maior aprofundamento relativo à associação de EA e OA, para que seja possível adotar tal estratégia em diversas pesquisas em IE, como, por exemplo, em Sistemas de Recomendação Educacionais - recomendando para os alunos os OA com atributos (tipo de interatividade expositivo, estrutura em rede, etc.) que melhor se adaptem aos seus EA (reflexivo, global, etc.).

Embora existam mais pesquisas que tenham realizado associações entre EA e OA, este estudo preferiu focar em apenas duas, recentes e no contexto brasileiro, devido à limitação de extensão do artigo, uma vez que a comparação teria que ser mais simplificada se fossem incorporados mais trabalhos a se comentar. Além disso, detalhando as diferenças entre duas abordagens, foi possível frisar a ideia de que, apesar de estar em alta o uso do conceito de EA, alguns aspectos não são de trivial entendimento e podem gerar visões diferenciadas.

Com base no entendimento da teoria de Felder-Silverman, os autores deste artigo discordam de alguns aspectos da abordagem de Oliveira (2013), a exemplo da associação entre Nível de Interatividade e a dimensão Compreensão, e da associação entre alguns Tipos de Recurso de Aprendizagem e a dimensão Processamento. Comentários neste nível de análise não foram realizados por não ser o enfoque deste artigo, mas este aspecto reforça a problemática de que, embora alguns pontos da teoria de Felder-Silverman possam ser facilmente entendidos e associados a atributos LOM, há questões que requerem mais estudos.

É interessante, portanto, que sejam fomentados estudos em parceria com estudiosos da teoria de Felder-Silverman (e demais teorias relativas a EA), como pesquisadores da área de educação e/ou psicologia, para que a parte psico-pedagógica fique melhor embasada e seja possível validar as propostas de ferramentas/técnicas computacionais baseadas nesses aspectos teóricos.

Quanto a isso, é apontado no relatório da SBC que os Grandes Desafios em Computação no Brasil exigem cooperação de pesquisadores da área da Computação com pesquisadores de várias outras áreas do conhecimento (SBC, 2006). Em relação ao desafio específico de "acesso participativo e universal do cidadão brasileiro ao conhecimento", tal relatório enfatiza que "várias áreas da Computação precisam contribuir para a solução deste problema", além de afirmar que "os benefícios da pesquisa nesta área atingem a coletividade, que pode reduzir a distância que temos hoje do maior bem da sociedade contemporânea: a informação, o conhecimento." (SBC, 2006).

Como trabalho futuro, pretende-se expandir esta comparação com outras pesquisas da literatura. Além disso, é interessante considerar outros modelos de EA também utilizados em IE para realizar análises similares.

Almeja-se, com a divulgação desta análise comparativa, motivar pesquisadores em IE a realizarem pesquisas associando o conceito de OA ao conceito de EA, uma vez que é importante considerar as particularidades dos estudantes no processo de ensinoaprendizagem. Além disso, os autores deste artigo reforçam a sugestão de realizar parcerias com estudiosos das áreas de, por exemplo, pedagogia e psicologia, para definirem em conjunto o que pode ser mais útil para alunos com determinadas características. 


\section{Agradecimentos}

À CAPES, pelo apoio financeiro, e aos pesquisadores que foram contatados, pelas informações disponibilizadas.

\section{Referências}

AGUIAR, J. J. B.; FECHINE, J. M.; COSTA, E. (2014). Estilos Cognitivos e Estilos de Aprendizagem em Informática na Educação: um mapeamento sistemático focado no SBIE, WIE e RBIE. Anais do XXV Simpósio Brasileiro de Informática na Educação (SBIE), Dourados, p. 441-450. Disponível em: <http://www.brie.org/pub/index.php/sbie/article/view/2972/2705>. Acesso em: 20 mai. 2015.

BRAGA, J.; PIMENTEL, E.; MENEZES, L.; DOTTA, S. (2014). Desafios sobre a Avaliação dos Ganhos de Aprendizagem na Educação mediada por TICs. Anais do XXXIV Congresso da Sociedade Brasileira de Computação (CSBC) - III Workshop de Desafios da Computação Aplicada à Educação (DesafIE), p. 670-678, Brasília, DF.

CARVALHO, V. (2015). RE: Protótipo de sistema de recomendação de OA [mensagem pessoal]. Mensagem recebida por <janderson@copin.ufcg.edu.br> em 12 de fevereiro de 2015.

CARVALHO, V. C.; DORÇA, F. A. (2014). Uma Abordagem para Recomendação Automática e Dinâmica de Objetos de Aprendizagem Baseada em Estilos de Aprendizagem e em Metadados no padrao IEEE LOM. Anais do XXXIV Congresso da Sociedade Brasileira de Computação (CSBC) - III Workshop de Desafios da Computação Aplicada à Educação (DesafIE), p. 642-651, Brasília, DF. Disponível em:

$<$ http://www.facom.ufu.br/ fabiano/publicacoes_arquivos/ArtigoDesafie2014.pdf>. Acesso em: 20 mai. 2015.

CARVALHO, V. C.; DORÇA, F. A.; CATTELAN, R. G.; ARAÚJO, R. D. (2014). Uma Abordagem para Recomendação Automática e Dinâmica de Objetos de Aprendizagem Baseada em Estilos de Aprendizagem. Anais do XXV Simpósio Brasileiro de Informática na Educação - SBIE 2014, p. 1188-1197, Dourados, MS. Disponível em: <http://www.br-ie.org/pub/index.php/sbie/article/view/3065/2573>. Acesso em: 20 mai. 2015.

COFFIELD, F.; MOSELEY, D.; HALL, E.; ECCLESTONE, K. (2004). Should we be using learning styles? What research has to say to practice. London, Learning and Skills Research Centre, Learning and Skills Development Agency. Disponível em: $<$ http://www.itslifejimbutnotasweknowit.org.uk/files/LSRC_LearningStyles.pdf $>$. Acesso em: 20 mai. 2015.

DIAS, C. C. L.; GASPARINI, I.; KEMCZINSK, A. (2009). Identificação dos estilos cognitivos de aprendizagem através da interação em um Ambiente EAD. In: XXIX Congresso da Sociedade Brasileira de Computação - XVII Workshop sobre Educação em Informática (WEI), p. 489-498. Disponível em: $<$ http://www.lbd.dcc.ufmg.br/colecoes/wei/2009/011.pdf>. Acesso em: 20 mai. 2015.

FELDER, R. M. (2002). Author's Preface - June 2002. In: FELDER, R. M.; SILVERMAN, L. K. Learning and Learning and Teaching Styles in Engineering 
Education. Disponível em: <http://www.ncsu.edu/felder-public/Papers/LS1988.pdf>. Acesso em: 20 mai. 2015.

FELDER, R. M.; SILVERMAN, L. K. (1988). Learning and Teaching Styles in Engineering Education. Journal of Engineering Education, v. 78, n. 7, p. 674-681. Disponível em: <http://www.ncsu.edu/felder-public/Learning_Styles.html>. Acesso em: 20 mai. 2015.

IEEE. (2002). IEEE Standard for Learning Object Metadata. IEEE Std 1484.12.1-2002, p. i-32.

KOLB, D. A. (1984). Experiential learning: experience as the source of learning and development. Englewood Cliffs: Prentice-Hall.

MACHION, A. C. G. (2007). Uso de ontologias e mapas conceituais na descoberta e análise de objetos de aprendizagem: um estudo de caso em eletrostática. 2007. Tese (Doutorado em Ciência da Computação) - Instituto de Matemática e Estatística, Universidade de São Paulo, São Paulo.

OLIVEIRA, I. C. A. (2013). AdaptMLearning: uma proposta de sistema de aprendizagem adaptativo e inteligente. Tese (Doutorado em Sistemas Eletrônicos) Escola Politécnica, Universidade de São Paulo - USP, São Paulo, 2013. Disponível em: $\quad<$ http://www.teses.usp.br/teses/disponiveis/3/3142/tde-26122013-150826/>. Acesso em: 20 mai. 2015.

RESENDE, D. T; DORÇA, F. A.; CATTELAN, R. G.; ARAÚJO, R. D. (2014). Em direção à recuperação automática de objetos de aprendizagem em repositórios através da associação dos estilos de aprendizagem de estudantes com metadados no padrão IEEE-LOM. Anais do VI Workshop Brasileiro de Web Semântica e Educação (WSWEd), Dourados, MS. Disponível em: <http://www.brie.org/pub/index.php/wcbie/article/view/3265/2805> . Acesso em: 20 mai. 2015.

RIDING, R.; RAYNER, S. (2000). Cognitive styles and learning Strategies understanding style differences in learning and behavior. London: David Fulton Publishers.

SBC. (2006). Grandes Desafios da Pesquisa em Computação no Brasil - 2006 - 2016. Relatório sobre o Seminário realizado em 8 e 9 de maio de 2006. Sociedade Brasileira de Computação. Disponível em: $<$ http://www.gta.ufrj.br/rebu/arquivos/SBC-Grandes.pdf>. Acesso em: 20 mai. 2015.

VALASKI, J.; MALUCELLI, A.; REINEHR, S. (2011). Revisão dos Modelos de Estilos de Aprendizagem Aplicados à Adaptação e Personalização dos Materiais de Aprendizagem. Anais do XXII Simpósio Brasileiro de Informática na Educação (SBIE), p. 844-847. Disponível em: <http://www.brie.org/pub/index.php/sbie/article/view/1843/1605>. Acesso em: 20 mai. 2015.

VAZ, M. Os Padrões Internacionais para a Construção de Material Educativo On-line. Pearson Education do Brasil, São Paulo, 1.ed., p. 386-396, 2009.

VICARI, R. M., RIBEIRO, A., DA SILVA, J. M. C., SANTOS, E. R., PRIMO, T., BEZ, M. (2010). Brazilian proposal for agent-based learning objects metadata standard - OBAA. Communications in Computer and Information Science, v. 108, p. $300-311$. 